\title{
Conformation of substituted poly-norbornene polymers studied by hyper-Rayleigh scattering at $1064 \mathrm{~nm}$
}

\author{
Chia-Chen Hsu ${ }^{\text {a, * }}$, Tzer-Hsiang Huang ${ }^{\text {a }}$, Sean Liu ${ }^{a}$, Fen-Fen Yeh ${ }^{a}$, Bih-Yaw Jin ${ }^{b}$, \\ Jitendra A. Sattigeri ${ }^{b}$, Chung-Wai Shiau ${ }^{b}$, Tien-Yau Luh ${ }^{b}$

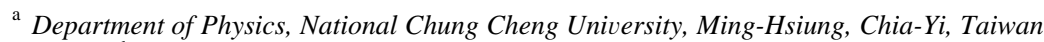 \\ ${ }^{\mathrm{b}}$ Department of Chemistry, National Taiwan University, Taipei, Taiwan
}

Received 1 March 1999; in final form 19 July 1999

\begin{abstract}
Hyper-Rayleigh scattering with a conventional $1064 \mathrm{~nm}$ laser source is employed to study the conformation of substituted poly-norbornene polymers that contain the non-linear optical chromophores as pendant groups. The dispersion-free first-hyperpolarizabilities $\left(\beta_{0}\right)$ of the polymers and pendant chromophores are determined and compared. A large enhancement of the $\beta_{0}$ values for the polymer samples is observed. Each of the chromophores is found to contribute $39 \%$ of its $\beta_{0}$ value to that of the polymer, in close agreement with the result of a simple calculation. This agreement supports the syndiotactic polymer conformation already predicted with the open-force-field calculation. Two-photon absorption-induced fluorescence (TPF) exists for most monomers but not for polymers in this study. The absence of TPF in the polymers may be due to the excited-state electronic interaction between pendant chromophores. (C) 1999 Elsevier Science B.V. All rights reserved.
\end{abstract}

Hyper-Rayleigh scattering (HRS) has been shown to be a useful technique for determining the first hyperpolarizability ( $\beta$ ) of non-linear optical (NLO) chromophores [1-4]. It is more straightforward than the electric-field-induced second-harmonic generation (EFISH) technique. It can also be applied to the ionic chromophores and the octopolar molecules to which EFISH cannot be applied [5]. Recently, Kauranen et al. have applied HRS (also EFISH) to investigate the second-order optical non-linearity of polymers which contain NLO chromophores as side

\footnotetext{
* Corresponding author. Tel.: + 886-5-2720411-6072; fax: +886-5-27-20-587; e-mail: cchsu@phy.ccu.edu.tw
}

groups [6,7]. The $\beta$ values of the polymers were enhanced with respect to those of free NLO chromophores. This supports the prediction that the polymer backbone is very rigid and the NLO chromophores are attached to the polymer backbone in a non-centrosymmetric arrangement. Furthermore, the NLO chromophores are orientationally correlated and hence contribute coherently to the $\beta$ value of the polymers $[6,7]$. On the contrary, no $\beta$ enhancement was reported for NLO chromophores incorporated in the polymer with a flexible backbone [8]. These results show that the rigidity of the polymer backbone is essential to the $\beta$ enhancement. As shown by Kauranen et al., HRS cannot only be used to determine the $\beta$ values of the NLO chromophores 
but can also be used to investigate the rigidity of the polymer backbone and the orientational correlation of pendant NLO chromophores.

Recently, we employed HRS with laser wavelengths longer than $1064 \mathrm{~nm}$ to measure and compare the dispersion-free first hyperpolarizabilities $\left(\beta_{0}\right)$ of the NLO chromophores and the substituted poly-norbornene (SPN) polymers that contain NLO chromophores as side groups [9]. Large $\beta_{0}$ enhancements for the polymer samples were observed. The enhancement was linearly proportional to the number of pendant chromophores. This further supports the polymer-chromophore structure mentioned above. We also estimated the conformation of the SPN polymer using HRS along with the semi-empirical molecular orbital (MO) and open-force-field (OFF)

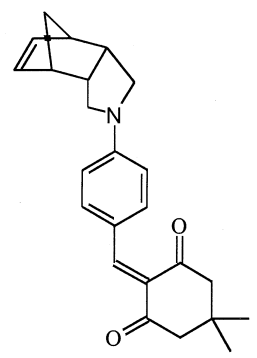

1a
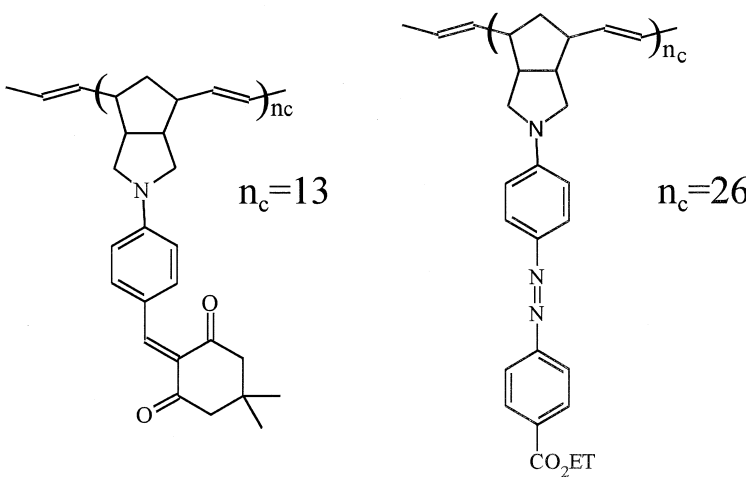

$2 \mathrm{a}$

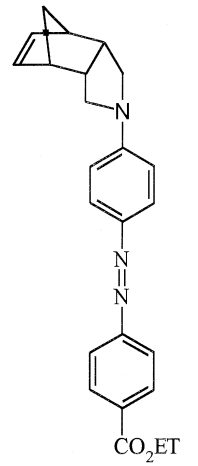

$1 b$

1c

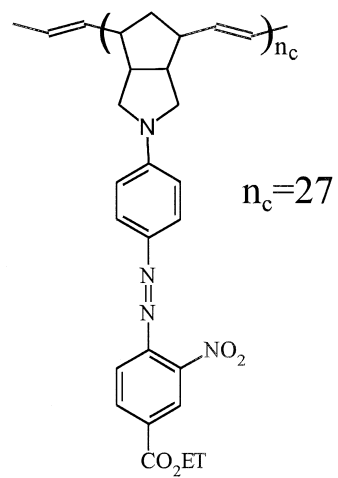

$2 c$

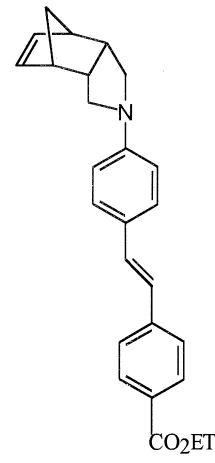

1d

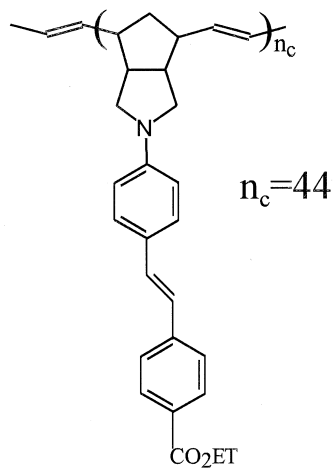

$2 d$

Fig. 1. Chemical structures of the eight molecules studied in this work. $n_{\mathrm{c}}$ is the number of chromophores $(1 \mathrm{a}-\mathrm{d})$ in the polymers $(2 \mathrm{a}-\mathrm{d})$. 
with the long-wavelength HRS supported the theoretically predicted syndiotactic conformation [9]. In this work, we employed the conventional short-wavelength $(1064 \mathrm{~nm}) \mathrm{HRS}$ to measure the $\beta$ values of the polymers and their pendant chromophores. The result is in line with our previous conclusion about the conformation of the SPN polymers. Furthermore, a calculation based on a simplified structural model for the syndiotactic polymer was performed to support experimental observation.

Fig. 1 shows the chemical structures of the samples studied. Samples 1a-d, are the substituted norbornene monomers. Samples $2 \mathrm{a}-\mathrm{d}$, are the SPN polymers that have in order $13,26,27$, and 44 , repeating units of the monomers shown above. The simplified structure of the syndiotactic polymer is shown in Fig. 2, where we assume the polymer backbone is stretched along the $z$-axis and the sidechain chromophores are all perpendicularly attached to the polymer backbone $\left(\theta=90^{\circ}\right)$ and uniformly distributed, i.e. $-60^{\circ}<\phi<60^{\circ}$. $\mathrm{CHCl}_{3}$ was the solvent used in this work. A solution of $p \mathrm{NA}$ (paranitroaniline) in $\mathrm{CHCl}_{3}$ was used as an external reference $\left(\beta_{p \mathrm{NA}}=23 \times 10^{-30} \mathrm{esu}\right)[1,2]$. The experimental details have been described elsewhere $[3,4]$.

The HRS intensity $I_{2 \omega}$ from a two-component solute-solvent system at very low concentration is proportional to the square of the incident laser intensity $I_{\omega}[1,2]$,

$I_{2 \omega}=G\left[N_{1}\left\langle\beta_{1}^{2}\right\rangle+N_{2}\left\langle\beta_{2}^{2}\right\rangle\right] I_{\omega}^{2} \exp \left(-\varepsilon N_{2} l\right)$.

In this equation \langle\rangle stands for orientational average. Subscripts ' 1 ' and ' 2 ' stand for solvent and solute, respectively. $N$ refers to the number density. $G$ is a constant related to the scattering geometry, local field correction factors at $\omega$ and $2 \omega$, and other instrument factors. The exponential term describes

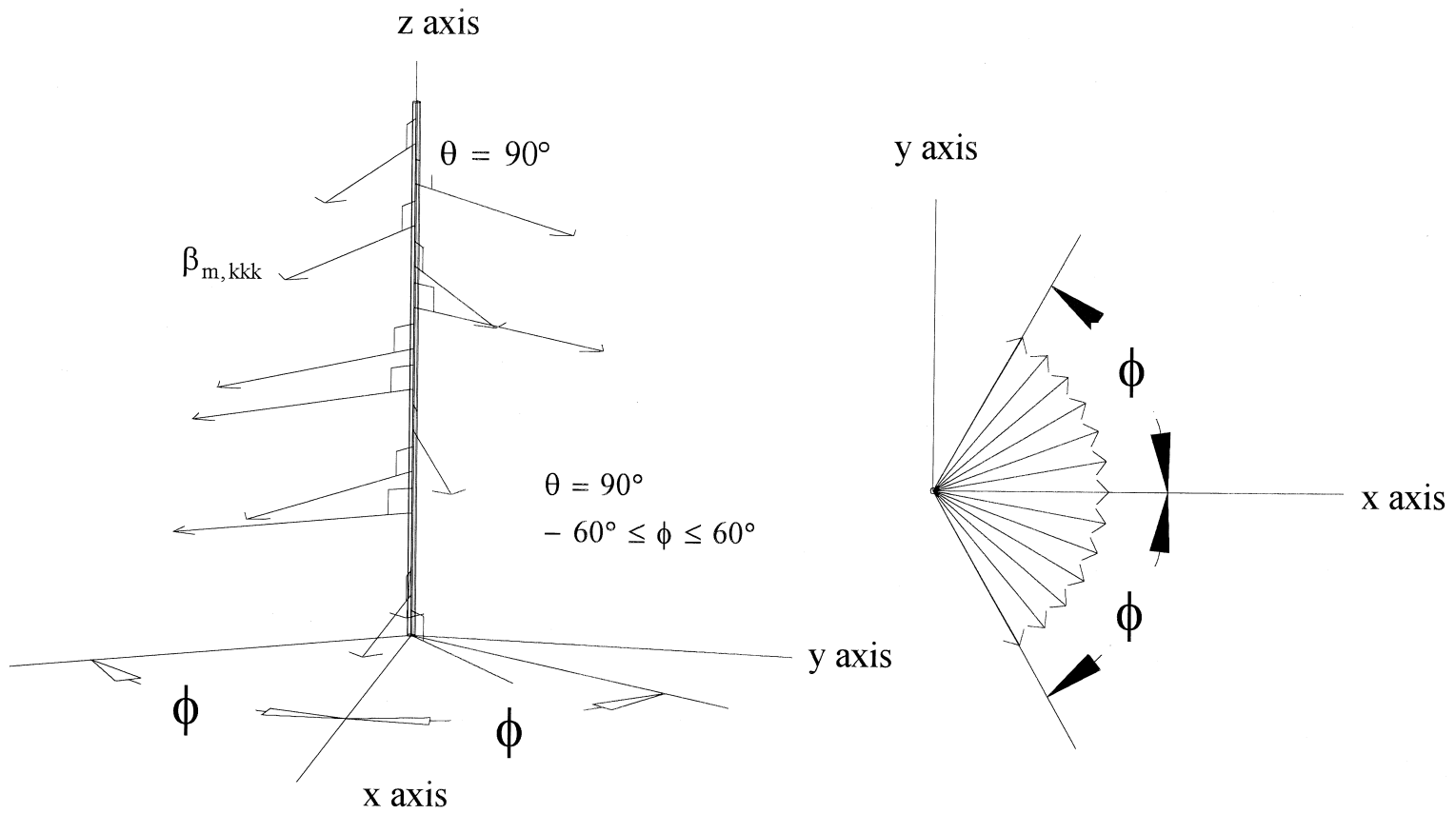

Viewed from the side of polymer backbone

Viewed along polymer backbone

Fig. 2. The theoretical syndiotactic conformation of poly-norbornene polymer. The polymer backbone follows the $z$-axis. The arrows denote $\beta_{\mathrm{m}, k k k}$ tensors of side-chain chromophores. 
the re-absorption of the HRS signal. $\varepsilon\left(1 \mathrm{~mol}^{-1}\right.$ $\mathrm{cm}^{-1}$ ) is the extinction coefficient of the sample solution at the second harmonic wavelength and $l$ is the effective path length that the $I_{2 \omega}$ light travels in the partially absorbing solution. Considering the incident laser beam as propagating along the laboratory $X$-axis with $Z$ polarization, the HRS signal is collected in the $Y$-axis with no polarization preference. For the solute and solvent, the orientational average of the $\beta$ squared can thus be written as

$$
\left\langle\beta^{2}\right\rangle=\left\langle\beta_{Z Z Z}^{2}\right\rangle+\left\langle\beta_{X Z Z}^{2}\right\rangle \text {. }
$$

The orientational average of the $\beta$ squared in the laboratory frame can be transformed to the molecular frame as follows $[10,11]$,

$$
\begin{aligned}
\left\langle\beta_{Z Z Z}^{2}\right\rangle= & \frac{1}{7} \sum_{i} \beta_{i i i}^{2}+\frac{6}{35} \sum_{i \neq j} \beta_{i i i} \beta_{i j j}+\frac{9}{35} \sum_{i \neq j} \beta_{i i j}^{2} \\
& +\frac{6}{35} \sum_{i j k, c y c l i c} \beta_{i i j} \beta_{j k k}+\frac{12}{35} \beta_{i j k}^{2} \\
\left\langle\beta_{X Z Z}^{2}\right\rangle= & \frac{1}{35} \sum_{i} \beta_{i i i}^{2}-\frac{2}{105} \sum_{i \neq j} \beta_{i i i} \beta_{i j j} \\
& +\frac{11}{105} \sum_{i \neq j} \beta_{i i j}^{2}-\frac{2}{105} \sum_{i j k, c y c l i c} \beta_{i i j} \beta_{j k k} \\
& +\frac{8}{35} \beta_{i j k}^{2},
\end{aligned}
$$

where $i, j$, and $k$, are the Cartesian axes in the molecular frame. Considering molecules with a quasi-linear structure such as the reference sample $p \mathrm{NA}$ and the monomer samples $1 \mathrm{a}-\mathrm{d}$, the molecular first hyperpolarizability tensor can assume only one major component $\beta_{k k k}, k$ being the conjugation axis. For chromophores with a quasi-linear structure, the $\left\langle\beta^{2}\right\rangle$ obtained with the scattering geometry described above can thus be expressed as

$$
\begin{aligned}
& \left\langle\beta^{2}\right\rangle=\left\langle\beta_{Z Z Z}^{2}\right\rangle+\left\langle\beta_{X Z Z}^{2}\right\rangle, \\
& \left\langle\beta^{2}\right\rangle=\frac{6}{35} \beta_{K K K}^{2} .
\end{aligned}
$$

For molecules with different symmetry, terms other than $\beta_{k k k}^{2}$ in Eqs. (3) and (4) need be considered in order to obtain the transformation between the macroscopic $\left\langle\beta^{2}\right\rangle$ and the molecular first hyperpolarizability tensor components [10,11].

Since the HRS signal is partially absorbed by the samples, plotting $I_{2 \omega} / I_{\omega}^{2}$ as a function of the concen- tration of solute $\left(\mathrm{N}_{2}\right)$ leads to a curved line as shown in Fig. 3. After removing the absorption effect, a linear plot of $I_{2 \omega}$ versus $N_{2}$ with a slope of $m_{2}$ is obtained as illustrated in Fig. 3. According to Eq. (1), the slope $m_{2}$ can be written as

$m_{2}=G\left\langle\beta_{2}^{2}\right\rangle$.

In the external reference method, the HRS signal of a reference sample with a quasi-linear structure is used to determine the first-hyperpolarizability of the unknown sample. The unknown and the reference samples are dissolved in the same solvent and are measured separately under the same experimental conditions. As a result, the $G$ constant for both samples is identical. The first hyperpolarizability of the unknown sample measured with the HRS technique $\beta^{\text {HRS }}$ can then be determined from the firsthyperpolarizability $\beta_{\mathrm{r}, k k k}$ of the reference and the ratio of the slope of the sample to that of the reference, $\left(m_{2} / m_{\mathrm{r}}\right)$,

$\beta^{\mathrm{HRS}} \equiv\left(\beta_{\mathrm{r}, k k k}\right)\left(\frac{m_{2}}{m_{\mathrm{r}}}\right)^{1 / 2}=\left(\frac{35}{6}\right)^{1 / 2}\left\langle\beta_{2}^{2}\right\rangle^{1 / 2}$,

where the subscript ' $r$ ' stands for the reference sample and the subscript ' 2 ' stands for the solute (quasi-

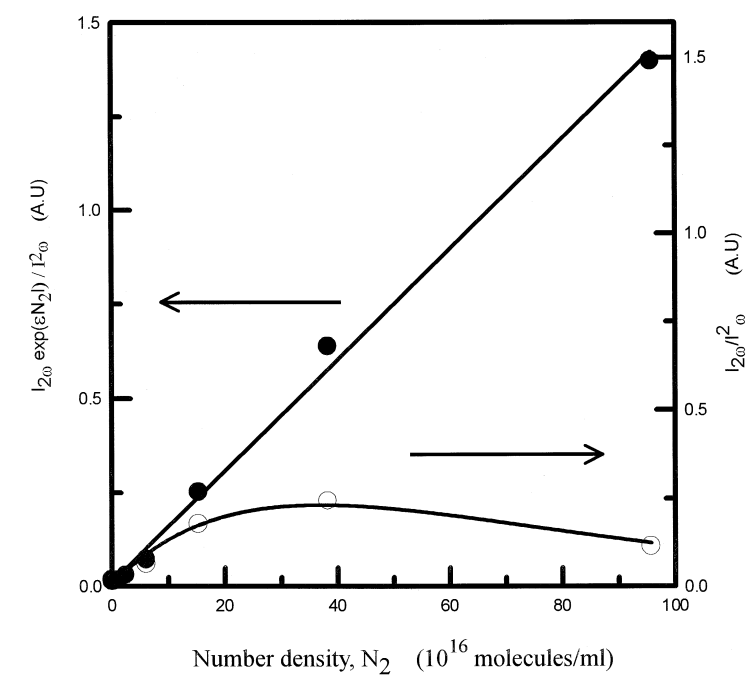

Fig. 3. Plots of corrected $I_{2 \omega} \exp \left(\varepsilon N_{2} l\right) / I_{\omega}^{2}$ and uncorrected $I_{2 \omega} / I_{\omega}^{2}$ versus the number density of sample $1 \mathrm{a}$ in $\mathrm{CHCl}_{3}$. The solid lines represent the theoretical fit to the experimental data points. 
linear chromophores or SPN polymers). In obtaining Eq. (8), we have applied Eq. (6) to the reference sample. For chromophores with a quasi-linear structure such as the monomer samples $1 \mathrm{a}-\mathrm{d}$, the first hyperpolarizability $\beta^{\mathrm{HRS}}$ is obtained by applying Eq. (6) to the monomers first and then combining with Eq. (8) to give,

$\beta_{\mathrm{m}}^{\mathrm{HRS}} \equiv\left(\beta_{\mathrm{r}, k k k}\right)\left(\frac{m_{\mathrm{m}}}{m_{\mathrm{r}}}\right)^{1 / 2}$,

$\beta_{\mathrm{m}}^{\mathrm{HRS}}=\left(\frac{35}{6}\right)^{1 / 2}\left\langle\beta_{\mathrm{m}}^{2}\right\rangle^{1 / 2}=\left(\beta_{\mathrm{m}, k k k}\right)$,

where ' $m$ ' stands for monomer. For the unknown samples with a non-linear structure such as the polymer samples $2 \mathrm{a}-\mathrm{d}$, we have $\beta^{\mathrm{HRS}}$ for the unknown polymer,

$\beta_{\mathrm{p}}^{\mathrm{HRS}} \equiv\left(\beta_{\mathrm{r}, k k k}\right)\left(\frac{m_{\mathrm{p}}}{m_{\mathrm{r}}}\right)^{1 / 2}$,

$\beta_{\mathrm{p}}^{\mathrm{HRS}}=\left(\frac{35}{6}\right)^{1 / 2}\left\langle\beta_{\mathrm{p}}^{2}\right\rangle^{1 / 2}$,

$\beta_{\mathrm{p}}^{\mathrm{HRS}}=\left(\frac{35}{6}\right)^{1 / 2}\left(\left\langle\beta_{\mathrm{p}, Z Z Z}^{2}\right\rangle+\left\langle\beta_{\mathrm{p}, X Z Z}^{2}\right\rangle\right)^{1 / 2}$,

where the subscript ' $p$ ' represents polymer and use has been made of Eq. (5) rather than Eq. (6) as in the case of monomers. The polymer first-hyperpolarizabilities in Eq. (10) are to be related to those of the pendant chromophores in the discussion below.

For all monomer and polymer samples studied in this Letter (Fig. 1), we summarize in Table 1 the first hyperpolarizabilities $\beta^{\mathrm{HRS}}$, measured with the 1064 and $1480 \mathrm{~nm}$ lasers, and their dispersion-free counterparts $\beta_{0}$ calculated from the un-damped two-state model. The $1480 \mathrm{~nm}$ data taken from Ref. [9] are repeated for comparison. Also shown in the table are the molecular weight, the number of chromophores in a polymer chain $n_{\mathrm{c}}$, and the absorption maximum $\lambda_{\max }$. The absorption spectra of the monomer and the polymer samples are similar although the polymer $\lambda_{\max }$ are slightly blue-shifted from those of the corresponding monomer samples. This has also been observed in other polymer systems with NLO chromophores attached to the side chains [6-8], and it agrees with the conjecture that the electron conjugation localizes in the side-chain NLO chromophores of the polymers, from there the optical non-linearity mainly arises. As such the non-linearity contribution from the polymer backbone can be neglected. As shown in Table 1 , the $\beta^{\mathrm{HRS}}(1064 \mathrm{~nm})$ values are all much larger than the $\beta^{\mathrm{HRS}}(1480 \mathrm{~nm})$ values. This is attributed to more favorable one- and two-photon resonance enhancements for the $1064 \mathrm{~nm}$ experiment. After correcting for resonance enhancements,

Table 1

First hyperpolarizabilities ( $\beta^{\mathrm{HRS}}$, measured; $\beta_{0}$, dispersion free) of the monomer and polymer samples studied

\begin{tabular}{|c|c|c|c|c|c|c|c|}
\hline \multirow[t]{2}{*}{ Sample } & \multirow{2}{*}{$\begin{array}{l}M_{\mathrm{w}}(\mathrm{PDI}) \\
(\mathrm{g} / \mathrm{mol})\end{array}$} & \multirow[t]{2}{*}{$n_{\mathrm{c}}$} & \multirow{2}{*}{$\begin{array}{l}\lambda_{\max }{ }^{a} \\
(\mathrm{~nm})\end{array}$} & \multicolumn{2}{|c|}{$\beta^{\mathrm{HRS}}\left(10^{-30} \mathrm{esu}\right)$} & \multicolumn{2}{|c|}{$\beta_{0}\left(10^{-30} \mathrm{esu}\right)$} \\
\hline & & & & $(1480 \mathrm{~nm})^{\mathrm{b}}$ & $(1064 \mathrm{~nm})$ & $(1480 \mathrm{~nm})^{\mathrm{c}}$ & $(1064 \mathrm{~nm})^{\mathrm{d}}$ \\
\hline $1 \mathrm{a}$ & 313 & & 475 & 59 & 211 & 31 & 34 \\
\hline $1 b$ & 387 & & 450 & $108^{\mathrm{e}}$ & 416 & $54^{\mathrm{e}}$ & 97 \\
\hline $2 b$ & $10000(1.9)$ & 26 & 435 & $874^{\mathrm{e}}$ & 1525 & $459^{e}$ & 421 \\
\hline $1 \mathrm{c}$ & 432 & & 495 & 65 & 758 & 32 & 80 \\
\hline $2 d$ & $17000(1.3)$ & 44 & 384 & 726 & 1146 & 459 & 478 \\
\hline
\end{tabular}

$M_{\mathrm{w}}$ is molecular weight, $n_{\mathrm{c}}$ is the number of chromophores attached to the polymer chain, and $\lambda_{\max }$ is the wavelength of the absorption maximum.

${ }^{\mathrm{a}} \mathrm{In} \mathrm{CHCl}_{3}$ solution.

${ }^{\mathrm{b}}$ From Ref. [9], measured at $1480 \mathrm{~nm}$.

${ }^{c}$ From Ref. [9], determined from the $\beta^{\text {HRS }}$ measured at $1480 \mathrm{~nm}$.

${ }^{\mathrm{d}}$ Determined from the $\beta^{\mathrm{HRS}}$ measured at $1064 \mathrm{~nm}$.

${ }^{\text {e }}$ From Ref. [9], measured at $1360 \mathrm{~nm}$. 
the $\beta_{0}(1064 \mathrm{~nm})$ and $\beta_{0}(1480 \mathrm{~nm})$ values become close except for samples $1 \mathrm{~b}-\mathrm{d}$. The larger $\beta_{0}$ (1064 $\mathrm{nm}$ ) values of monomer samples $1 \mathrm{~b}-\mathrm{d}$ are caused by the two-photon absorption induced fluorescence (TPF) [12]. The $\beta_{0}(1064 \mathrm{~nm})$ and $\beta_{0}(1480 \mathrm{~nm})$ values of the polymer samples are close, and it indicates that the TPF contribution to HRS disappears in polymers with correlated chromophores. This observation is different from a previous study with a flexible polymer backbone [8]. The absence of TPF in the polymers may be due to the excited-state electronic interaction between pendant chromophores. Since the linear absorption spectra of the monomer and polymer are similar, no electronic interaction in the ground state can be assumed. However, the excited-state electronic interaction may decrease the population of the excited state and suppress the TPF intensity of the polymers. Without the TPF contribution, $\beta_{0}(1064 \mathrm{~nm})$ will generally agree with the $\beta_{0}(1480 \mathrm{~nm})$ indicating that the un-damped two-state model is appropriate for the measured samples. Even with the TPF contribution, the $\beta_{0}$ (1064 $\mathrm{nm}$ ) values of the monomer samples are still unambiguously smaller than those of the polymer samples. Note that samples $1 \mathrm{a}$ and $2 \mathrm{a}$ both have no TPF and the $\beta_{0}$ of $2 \mathrm{a}$ is enhanced five times with respect to that of 1a. The $\beta_{0}$ enhancement for the polymer samples help us to deduce the same conclusions that we drew in our previous work [9]. That is: (1) the conformation of the substituted poly-norbornenes is syndiotactic, (2) the backbone of the substituted poly-norbornenes is rigid, and (3) the pendant sidegroup chromophores are orientationally correlated, and each chromophore contributes coherently to the first hyperpolarizability of the polymer. From this experimental result of samples $1 \mathrm{a}$ and $2 \mathrm{a}$, the $\beta_{\mathrm{p}}^{\mathrm{HRS}}$ can be written as

$\beta_{\mathrm{p}}^{\mathrm{HRS}}=0.39 n_{\mathrm{c}} \beta_{\mathrm{m}, k k k}$.

This indicates that each of the pendant chromophores contributes $39 \%$ of its monomeric value to that of the polymer, which agrees with our previous observation using the long-wavelength HRS technique [9].

Since the non-linearity contribution is only from the pendant NLO chromophores, the first hyperpolarizability of the polymer can be calculated from the vector sum of all contributions from the chromophores. Consider the simple theoretical model of the syndiotactic conformation for the polymer as illustrated in Fig. 2. By projecting the $\beta_{\mathrm{m}, k k k}$ tensor of side-chain chromophores onto the polymeric coordinate and vector - summing over all of them, the non-vanishing polymeric $\beta$ tensors follows

$$
\begin{aligned}
\beta_{y y x} & =n_{\mathrm{c}} \beta_{\mathrm{m}, k k k} \frac{\int_{-\pi / 3}^{\pi / 3} \sin ^{2} \phi \cos \phi \mathrm{d} \phi}{\int_{-\pi / 3}^{\pi / 3} \mathrm{~d} \phi} \\
& =0.21 n_{\mathrm{c}} \beta_{\mathrm{m}, k k k}, \\
\beta_{x x x} & =n_{\mathrm{c}} \beta_{\mathrm{m}, k k k} \frac{\int_{-\pi / 3}^{\pi / 3} \cos ^{3} \phi \mathrm{d} \phi}{\int_{-\pi / 3}^{\pi / 3} \mathrm{~d} \phi}=0.62 n_{\mathrm{c}} \beta_{\mathrm{m}, k k k} .
\end{aligned}
$$

Using the Kleinman symmetry and substituting Eqs. (12) and (13) to Eqs. (3) and (4), we obtain

$$
\begin{aligned}
\left\langle\beta_{\mathrm{p}, Z z Z}^{2}\right\rangle= & \frac{1}{7} \beta_{x x x}^{2}+\frac{6}{35} \beta_{x x x} \beta_{x y y} \\
& +\frac{9}{35} \beta_{y y x}^{2}\left\langle\beta_{\mathrm{p}, Z z z}^{2}\right\rangle \\
= & 0.088 n_{\mathrm{c}}^{2} \beta_{\mathrm{m}, k k k}^{2}, \\
\left\langle\beta_{p, X z Z}^{2}\right\rangle= & \frac{1}{35} \beta_{x x x}^{2}-\frac{2}{105} \beta_{x x x} \beta_{x y y}+\frac{11}{105} \beta_{y y x}^{2}, \\
= & 0.013 n_{\mathrm{c}}^{2} \beta_{\mathrm{m}, k k k}^{2} .
\end{aligned}
$$

Substituting Eqs. (14) and (15) to Eq. (10), the $\beta_{\mathrm{p}}^{\mathrm{HRS}}$ of the polymers is found to be

$\beta_{\mathrm{p}}^{\mathrm{HRS}}=0.76 n_{\mathrm{c}} \beta_{\mathrm{m}, k k k}$.

As shown by Eq. (16) each monomer contributes $76 \%$ of its $\beta_{0}$ value to that of the polymer, which is about twice the experimental observation. That the calculated result is higher likely arises from the assumptions made. First, the polymer is assumed to be syndiotactic with a stretched out backbone structure, to which the perpendicularly attached linear chromophores are uniformly distributed between $-60^{\circ}$ and $60^{\circ}$. Second, we neglected the interaction between pendant chromophores, with which individ- 
ual chromophores would have contributed less to the polymer's first-hyperpolarizability. It is noticed that, for the poly-isocyanides polymer, the difference between experimental observation and theoretical prediction is somewhat larger [6]. Thus our assumption of the syndiotactic conformation of the SPN polymers is after all not unrealistic.

In conclusion, the conventional short-wavelength (1064 nm) HRS technique was used to study the conformation of the SPN polymers. The results agree with our previous observation [9] using the longwavelength HRS technique. The large $\beta$ enhancement on the SPN polymers, which we obtain both theoretically and experimentally, provides further support to the syndiotactic polymer conformation. The theoretical prediction of the enhancement, though based on a simple model, agrees reasonably well with the experimental observation. TPF exist for most monomers but not for polymers in this study. The absence of TPF in the polymers may be due to the excited-state electronic interaction between pendant chromophores.

This research was supported financially by the National Science Council of Taiwan under Contracts No. NSC 88-2112-M-194-013 (C.C.H.) and 882112-M-194-008 (T.H.H.). C.C.H. gratefully ac- knowledges fruitful discussions with Prof. C.H. Wang.

\section{References}

[1] K. Clays, A. Persoons, Phys. Rev. Lett. 66 (1991) 2980.

[2] K. Clays, A. Persoons, Rev. Sci. Instrum. 63 (1992) 3285.

[3] C.C. Hsu, T.H. Huang, Y.Z. Zang, J.L. Lin, Y.Y. Cheng, J.T. Lin, H.H. Wu, C.H. Wang, C.T. Kuo, C.H. Chen, J. Appl. Phys. 80 (1996) 5996.

[4] C.C. Hsu, C.F. Shu, T.H. Huang, C.H. Wang, J.L. Lin, Y.K. Wang, Y.L. Zang, Chem. Phys. Lett. 274 (1997) 446.

[5] T. Verbiest, K. Clays, A. Persoons, Opt. Lett. 18 (1992) 525.

[6] M. Kauranen, T. Verbiest, C. Boutton, M.N. Teerenstra, K. Clays, A.J. Schouten, R.J.M. Nolte, A. Persoons, Science 270 (1995) 965.

[7] T. Verbiest, C. Samyn, C. Boutton, S. Houbrechts, M. Kauranen, A. Persoons, Adv. Mat. 8 (1996) 756.

[8] O.K. Song, J.N. Woodford, C.H. Wang, J. Phys. Chem. 106 (1997) 2819

[9] J.A. Sattigeri, C.W. Shiau, C.C. Hsu, F.F. Yeh, S. Liu, B.Y. Jin, T.Y. Luh, J. Am. Chem. Soc. 121 (1999) 1607.

[10] S.J. Cyvin, J.E. Rauch, J.C. Decius, J. Chem. Phys. 43 (1965) 4083.

[11] R. Bersohn, Y.H. Pao, H.L. Frisch, J. Chem. Phys. 45 (1966) 3184.

[12] M.C. Flipse, R. de Jonge, R.H. Woundenberg, A.A. Marsman, C. van Walree, L.W. Jenneskens, Chem. Phys. Lett. 245 (1995) 297. 